\title{
Efficacy and Safety of 125I Seed Brachytherapy Combined with Gemcitabine Containing Chemotherapy for Unresectable Pancreatic Neoplasm-A Meta-Analysis
}

\author{
Liuyi Yang ${ }^{1,2}$, Sen Yang ${ }^{1,2}$, Huayue Cong ${ }^{1,2}$, Qiumei Zhou ${ }^{1,2}$, Aixia Sui ${ }^{1, *}$ \\ ${ }^{1}$ Department of Oncology, Hebei General Hospital, Shijiazhuang, Hebei, China \\ ${ }^{2}$ Graduate School of North China University of Science and Technology, Tangshan, Hebei, China \\ *Correspondence Author, suiaxhebei@126.com
}

\begin{abstract}
These instructions provide you guidelines for preparing papers for Journal of Innovation and Social Science Research. Use this document as a template and as an instruction set. PURPOSE: The goal of this meta-analysis was to look at the efficacy and safety of radioactive seeds brachytherapy using Iodine-125 for unresectable advanced pancreatic neoplasm. METHODS AND MATERIALS: In pancreatic neoplasm, trials comparing ${ }^{125}$ I brachytherapy with Gemcitabine chemotherapy have been identified. The pooled odds ratios for overall response rate $(O R R)$, disease control rate $(D C R)$, and complications, as well as the pooled hazard ratios for progression free survival (PFS) and overall survival, were calculated using meta-analysis (OS). RESULTS: A total of 12 studies with a total of 863 cases were included. There were significant differences in ORR (OR=3.13, 95\% CI 2.24-4.38) and DCR (OR=3.67, 95\% CI 2.63-5.12) between 125 I brachytherapy combined with chemotherapy containing gemcitabine and chemotherapy alone, but no statistical differences in PFS (HR=0.63, 95\% CI 0.30-1.32), OS (HR=0.72, $95 \%$ CI 0.43-1.20), and adverse reactions (diarrhea: $O R=0.75$, 95\%CI: 0.42-1.25; pernicious vomiting: $O R=1.30,95 \% C I$ : 0.82-2.06; leukopenia: $O R=1.32,95 \% C I$ : 0.88-1.98; thrombocytopenia: $O R=1.05$, 95\%CI: 0.71-1.57; anemia: OR=1.18, 95\%CI: 0.66-2.10; and hepatic impairment: OR=1.10.95\% CI: 0.63-1.92). CONCLUSION: ${ }^{125}$ I brachytherapy combined with the inclusion of gemcitabine chemotherapy enhance the clinical efficacy of patients with unresectable pancreatic neoplasm without increasing the incidence of adverse effects such as pancreatic fistula, pancreatitis, seed migration, and duodenitis compared with chemotherapy. However, there was no obvious effect on the survival of unresectable pancreatic neoplasm.
\end{abstract}

Keywords: Pancreatic neoplasm, Iodine-125, Brachytherapy, Gemcitabine, Efficacy, Meta-analysis.

\section{Introduction}

Pancreatic neoplasm is the seventh leading cause of cancer-related death and one of the deadliest tumors in the world [1-2]. At present, the cause of pancreatic neoplasm is unclear and most of pancreatic neoplasm is already in the advanced stage when symptoms appear, and there is no indication for surgery [3]. Nowadays, Gemcitabine is a standard treatment for pancreatic neoplasm and it is widely used in the first-line treatment of pancreatic neoplasm, single or combined use of gemcitabine has been proven to prolong the survival of patients with pancreatic neoplasm [4]. To make matters worse, the current available chemotherapy options are limited and often ineffective, especially for patients with primary diagnosis in the III/IV stage [5-7]. A Chinese clinical epidemiology statistics show that the 1-year overall survival (OS) rate of pancreatic neoplasm patients is $<25 \%$, and $23.0 \%$ of patients receiving other treatments (except surgery) have 3.0 months of median overall survival (mOS) [8]. Because pancreatic neoplasm cells exhibit multiple resistance mechanisms including genetic and epigenetic changes, complex and dense tumor microenvironment, and other factors, they exhibit significant resistance to chemotherapy, radiotherapy, and targeted therapy [9]. In the past decade, it has made relevant progress in diagnostic methods, perioperative management, radiotherapy techniques and systemic treatment of advanced diseases in pancreatic neoplasm, but only moderate progress has been made in patient prognosis [10]. However, Iodine-125 radioactive seed brachytherapy ( ${ }^{125}$ I brachytherapy) showed an accurate effect when it was first applied to the treatment of pancreatic neoplasm [11]. Meanwhile, previous studies have shown that patients treated with gemcitabine alone or combined chemotherapy containing gemcitabine have better OS, progression-free survival (PFS) and quality of life (QoL) [12]. However, because ${ }^{125} \mathrm{I}$ brachytherapy is not widely used in the treatment of unresectable pancreatic neoplasms, there have been few randomized clinical trials in recent years. As a result, the goal of this research is to compile and summarize evidence on the efficacy and safety of ${ }^{125} \mathrm{I}$ brachytherapy for unresectable advanced pancreatic neoplasm. In order to provide more latest evidence to guide future clinical trials, we compared the efficacy and safety of ${ }^{125} \mathrm{I}$ brachytherapy combined with chemotherapy regimen containing gemcitabine and chemotherapy regimen containing gemcitabine alone.

\section{Methods and Materials}

\subsection{Literature Search}

Without language constraints, PubMed, Cochrane Library, Embase, China national knowledge infrastructure (CNKI), and WANFANG MED ONLINE were searched to find relevant papers up to February 2021. The main keywords used for the search were "brachytherapy OR Iodine-125 OR ${ }^{125} \mathrm{I}$ OR implanted radiotherapy" AND "pancreatic neoplasms OR pancreatic neoplasm OR pancreas neoplasm" AND "Gemcitabine".

Studies inclusion and exclusion criteria 
The following were the key inclusion criteria: (1) The research was limited to controlled clinical trials, such as randomized controlled trials (RCTs) and non-RCTs. (2) Patients received Iodine-125 radioactive seeds brachytherapy combined with chemotherapy in the treatment group and chemotherapy only in the control group for the treatment of pancreatic neoplasm; (3) Patients with unresectable advanced pancreatic neoplasm diagnosed pathologically or clinically, no chemotherapy contraindication before treatment, and no obvious abnormalities in liver, kidney, or heart function; and (4) Studies had ORR and DCR outcomes defined by World Health Organization.

The following were the key exclusion criteria: (1) single-arm research; (2) animal experiments, reviews, and other irrelevant studies; (3) no specific data on primary outcomes from literature studies and connections with authors; (4) patients with resectable pancreatic neoplasm, and the control group did not receive intravenous or oral chemotherapy.

\subsection{Data Extraction and Quality Assessment}

According to the Preferred Reporting Items for Systematic Reviews and Meta-Analysis statement [13], two reviewers (LYY and SY) independently searched potentially relevant articles and conducted the data extraction, and any disagreements between the two reviewers were resolved consensually by involving a third reviewer (AXS). Each study's publication details, demographic and clinical information, and outcome variables such as ORR, DCR, OS, PFS, and complications were all collected.

The general methodological quality of the included RCTs was assessed according to the Cochrane Collaboration's Risk of Bias (ROB) criteria of Cochrane handbook [14]. Random sequence generation, allocation concealment, blinding of participants and personnel, blinding of outcome assessment, incomplete outcome data, selective reporting, and other bias were all used to assess the quality of each included RCT. Each item was categorized into three categories: high risk, unknown risk, and low risk. The Newcastle-Ottawa Scale (NOS) assessment instrument was used to assess the quality of non-RCTs [15]. Selection criteria included adequate case definitions, representativeness of the cases, selection of controls, definition of controls; comparability of cases and controls based on design or analysis; exposure criteria included exposure ascertainment, same method of ascertainment for cases and controls, and non-response rate.

\subsection{Curative Effects}

Complete response (CR), partial response (PR), stable disease (SD), and progressive disease (PD) were the markers for solid tumor responses, according to the World Health Organization and International Union Against Cancer [16]. CR denotes that all target lesions have vanished, no new lesions have formed, and tumor markers have been normal for at least four weeks. PR indicates that the sum of the target lesions' maximum diameters dropped by $30 \%$ and remained stable for at least 4 weeks. SD denotes that the sum of the target lesions' maximum diameters did not decrease to PR or increased to PD. PD indicates that the sum of the target lesions' maximum diameters has increased by at least $20 \%$, or that additional lesions have appeared. ORR, which equals $\mathrm{CR}+\mathrm{PR}$, and DCR, which equals $\mathrm{CR}+\mathrm{PR}+\mathrm{SD}$, are the major outcomes in our study. In this study, the complications of the treatment and control groups were assessed for clinical efficacy.

\subsection{Statistical Analysis}

Review Manager 5.3 was used to conduct the analysis. The hazard ratio (HR) was generated for measuring OS and PFS using the inverse variance approach, and the odds ratio (OR) was employed as the fundamental assessment of dichotomous data using the Mantel-Haenszel method. We combined an HR and its $95 \%$ confidence interval (CI) in each research to pool estimates of OS and PFS findings.

We calculated these factors from the given data using Tierney et al's methodology [17] if these variables were not available in an article. The $\mathrm{I} 2$ statistic and the p-value were used to assess statistical heterogeneity among the research involved. We utilized a fixed-effects technique with an $\mathrm{I} 2<50 \%$ or p $>0.1$ significant level; otherwise, we used a random-effect method. Funnel plots were used to test for publication bias. Finally, sensitivity analyses were performed to assess the robustness of the association results by systematically omitting one study at a time. If the pooled estimates were stable following the sensitivity analysis, it was likely that the findings were not influenced by a single study. Differences were considered statistically significant if $p<0.05$. Subgroup analysis or sensitivity analysis are employed if significant heterogeneity is discovered. If publishing bias was detected, the trim-and-fill procedure was applied to correct the asymmetry [18]. Finally, sensitivity analysis was used to assess the robustness of relevant results by omitting individual studies one by one at one iteration. If the pooled estimates stay steady after sensitivity analysis, it means the findings aren't influenced by a single study.

\section{Results}

\subsection{Characteristics of the Studies and Quality Assessment}

The progress of literature retrieval is shown in Figure 1. During the initial literature search, 117 records were discovered. A total of 86 studies were reviewed after duplicate studies were eliminated. After reading the title and abstract, we found that 63 studies did not match our criteria for inclusion. Eleven studies were eliminated after reading the entire text: 1 study population did not meet the requirements, 4 study intervention measures did not meet the requirements, 2 study outcome indicators did not meet the requirements, 1 study was a meeting report, 1 study had no clear results, and 2 studies did not meet our inclusion criteria in other aspects. In the meta-analysis, 12 studies including 863 patients were recruited after studies with inaccurate and unusable data were excluded [19-30]. Table 1 lists the features of the 12 studies. 




Figure 1: Flow diagram of the study selection process

Notes: CNKI=China Knowledge Resource Integrated Database

Table 1: Characteristic of the included studies

\begin{tabular}{|c|c|c|c|c|c|c|c|c|c|c|c|}
\hline \multirow{2}{*}{ Study } & \multirow{2}{*}{$\mathrm{N}$} & \multirow{2}{*}{$\begin{array}{c}\text { Gender } \\
(\mathrm{M} / \mathrm{F})\end{array}$} & \multirow{2}{*}{$\begin{array}{c}\text { Age } \\
\text { (average/range) }\end{array}$} & \multirow{2}{*}{$\begin{array}{c}\text { Tumor stage } \\
\text { conditions }\end{array}$} & \multirow{2}{*}{$\mathrm{T} / \mathrm{C}$} & \multicolumn{2}{|c|}{ Treatment } & \multicolumn{4}{|c|}{ Outcomes } \\
\hline & & & & & & $\mathrm{T}$ & $\mathrm{C}$ & ORR & DCR & OS & PFS \\
\hline $\mathrm{Yu}, 2014$ & 30 & $11 / 19$ & $\begin{array}{c}\mathrm{T}: 61.20 \pm 12.5(-) \\
\mathrm{C}: 59.47 \pm 10.62(-)\end{array}$ & III: 25 and IV:5 & $15 / 15$ & $\begin{array}{l}{ }^{125} \mathrm{I}+\mathrm{G}+\text { Extern } \\
\text { al radiotherapy }\end{array}$ & $\begin{array}{l}\mathrm{G}+\text { External } \\
\text { radiotherapy }\end{array}$ & $\mathrm{Y}$ & $\mathrm{Y}$ & $\mathrm{Y}$ & \\
\hline Shi,2017 & 82 & $53 / 29$ & $\begin{array}{c}\mathrm{T}: 49(39-73) \\
\mathrm{C}: 48.3(38-72)\end{array}$ & III:49 and IV:33 & $41 / 41$ & ${ }^{125} \mathrm{I}+\mathrm{GS}$ & GS & $\mathrm{Y}$ & $\mathrm{Y}$ & & \\
\hline Yang,2014 & 88 & $53 / 35$ & $50(-)$ & IV:40 & $45 / 43$ & ${ }^{125} \mathrm{I}+\mathrm{GP}$ & GP & $\mathrm{Y}$ & $\mathrm{Y}$ & $\mathrm{Y}$ & \\
\hline $\mathrm{Hu}, 2007$ & 64 & $24 / 30$ & $\begin{array}{l}\mathrm{T}: 62 \pm 10.64(46-75) \\
\mathrm{C}: 62 \pm 8.27(46-74)\end{array}$ & II:22 and III:42 & $32 / 32$ & ${ }^{125} \mathrm{I}+\mathrm{G}$ & G & $\mathrm{Y}$ & $\mathrm{Y}$ & & \\
\hline Luo,2021 & 66 & $45 / 21$ & 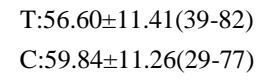 & III:23 and IV:43 & $35 / 31$ & ${ }^{125} \mathrm{I}+\mathrm{GP}$ & GP & $\mathrm{Y}$ & $\mathrm{Y}$ & $\mathrm{Y}$ & $\mathrm{Y}$ \\
\hline Yan,2019 & 98 & $61 / 37$ & $\begin{array}{l}\mathrm{T}: 48.25 \pm 5.32(39-80) \\
\mathrm{C}: 48.94 \pm 5.41(38-80)\end{array}$ & III and IV & $49 / 49$ & ${ }^{125} \mathrm{I}+\mathrm{GS}$ & GS & $\mathrm{Y}$ & $\mathrm{Y}$ & & \\
\hline Wang,2020 & 43 & $20 / 22$ & $\begin{array}{l}\mathrm{T}: 55.41 \pm 2.53(39-75) \\
\mathrm{C}: 55.61 \pm 2.41(40-76)\end{array}$ & III & $22 / 21$ & ${ }^{125} \mathrm{I}+\mathrm{GC}$ & GC & $\mathrm{Y}$ & $\mathrm{Y}$ & & \\
\hline Sun,2009 & 47 & $29 / 18$ & $-(40-83)$ & $\begin{array}{l}\text { T: III:17 and IV:8 } \\
\text { C: III:15 and IV:8 }\end{array}$ & $25 / 22$ & ${ }^{125} \mathrm{I}+\mathrm{GP}$ & GP & $\mathrm{Y}$ & $\mathrm{Y}$ & & \\
\hline $\mathrm{Li}, 2015$ & 51 & $35 / 19$ & $-(38-69)$ & $\begin{array}{c}\text { T: II:5, III: } 16 \text { and } \\
\text { IV:6 } \\
\text { C: II:7, III: } 13 \text { and } \\
\text { IV:4 }\end{array}$ & $27 / 24$ & ${ }^{125} \mathrm{I}+\mathrm{GP}$ & GP & $\mathrm{Y}$ & $\mathrm{Y}$ & $\mathrm{Y}$ & \\
\hline Huang,2020 & 143 & $81 / 62$ & $\begin{array}{l}\mathrm{T}: 61.65 \pm 11.224(44-81) \\
\mathrm{C}: 60.59 \pm 10.348(42-83)\end{array}$ & III and IV & $72 / 71$ & ${ }^{125} \mathrm{I}+\mathrm{GP}$ & GP & & & $\mathrm{Y}$ & $\mathrm{Y}$ \\
\hline $\mathrm{He}, 2018$ & 83 & $51 / 32$ & 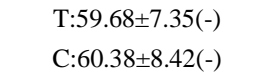 & $\begin{array}{l}\text { T: III:13 and IV:23 } \\
\text { C: III:18 and IV:29 }\end{array}$ & $36 / 47$ & ${ }^{125} \mathrm{I}+\mathrm{GS}$ & GS & $\mathrm{Y}$ & $\mathrm{Y}$ & & \\
\hline Lun, 2015 & 68 & $41 / 27$ & $-(39-80)$ & III:22 and IV:46 & $38 / 30$ & ${ }^{125} \mathrm{I}+\mathrm{GS}$ & GS & $\mathrm{Y}$ & $\mathrm{Y}$ & $\mathrm{Y}$ & $\mathrm{Y}$ \\
\hline
\end{tabular}


The methodological quality of RCTs was assessed using the Cochrane Collaboration's ROB criteria, while the methodological quality of non-RCTs was examined using the NOS assessment tool. Allocation concealment bias was shown to be riskier in five RCT studies, and three studies did not give a concrete randomized technique. Among the 7 non-RCT studies, 5 studies had 9 scores and the other two had 8 scores. All non-RCTs were high-quality studies. (The details are reported in Table 2). ROB

Table 2: Literature quality analysis

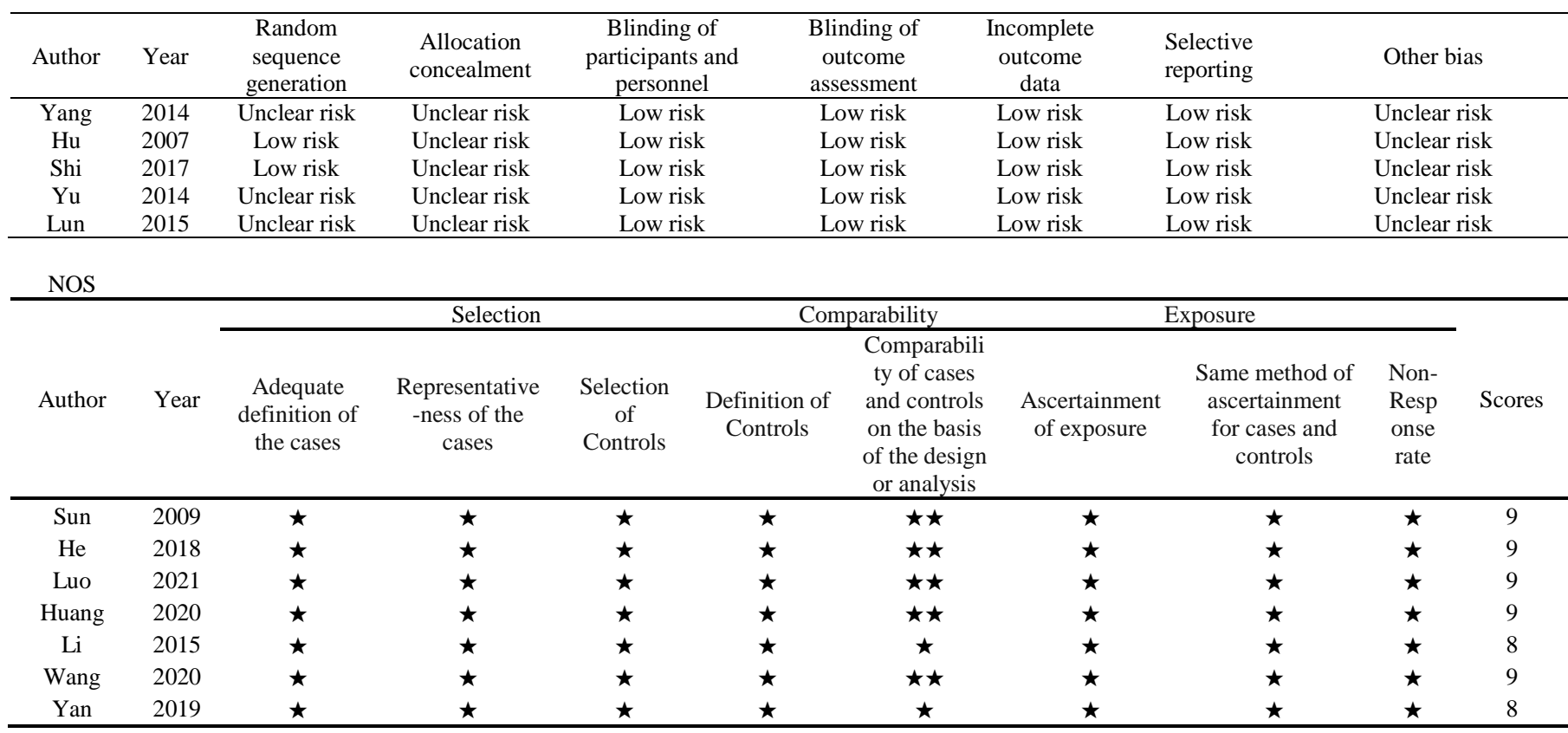

\subsection{Efficacy Assessment}

In the pooled analysis of ORR, the I2 test (I $2=14 \%$, $\mathrm{p}<0.00001)$ showed mild heterogeneity among the 11 studies. Meta-analysis showed that the ORR was significantly different between the two groups $(\mathrm{OR}=3.13,95 \% \mathrm{CI} 2.24-4.38)$ in the fixed effect model (Figure 2). Treatment groups use ${ }^{125} \mathrm{I}$ brachytherapy in combination with chemotherapy in these

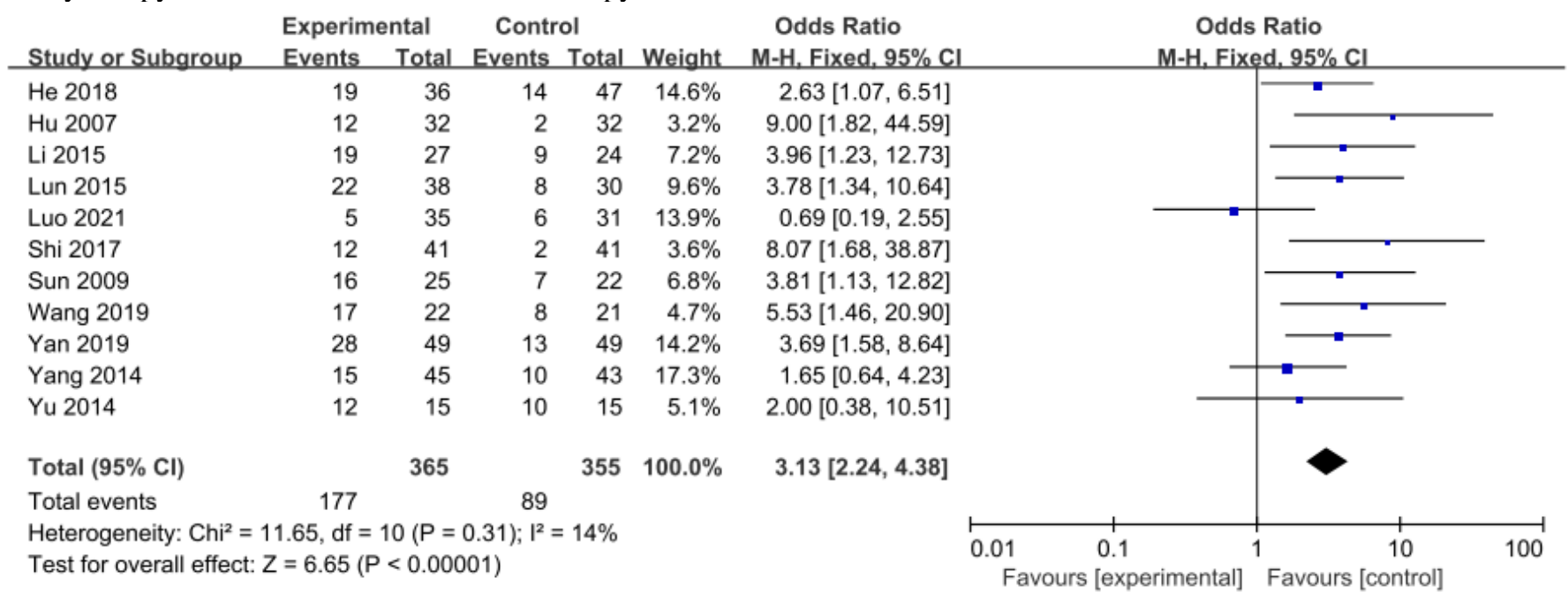

Figure 2: Forest plot of studies evaluating ORs of ORR studies, while the control group only uses chemotherapy. Eleven studies were included in the pooled analysis of DCR. The I2 test $(\mathrm{I} 2=45 \%, \mathrm{p}<0.0001)$ showed moderate heterogeneity among these studies. Meta-analysis showed that there were significant differences in DCR between the two groups $(\mathrm{OR}=3.67,95 \% \mathrm{CI} 2.63-5.12)$ in the fixed effect model (Figure 3).

Notes: $\mathrm{CI}=$ confidence interval; $\mathrm{OR}=$ odd ratio; $\mathrm{ORR}=$ overall response rate 


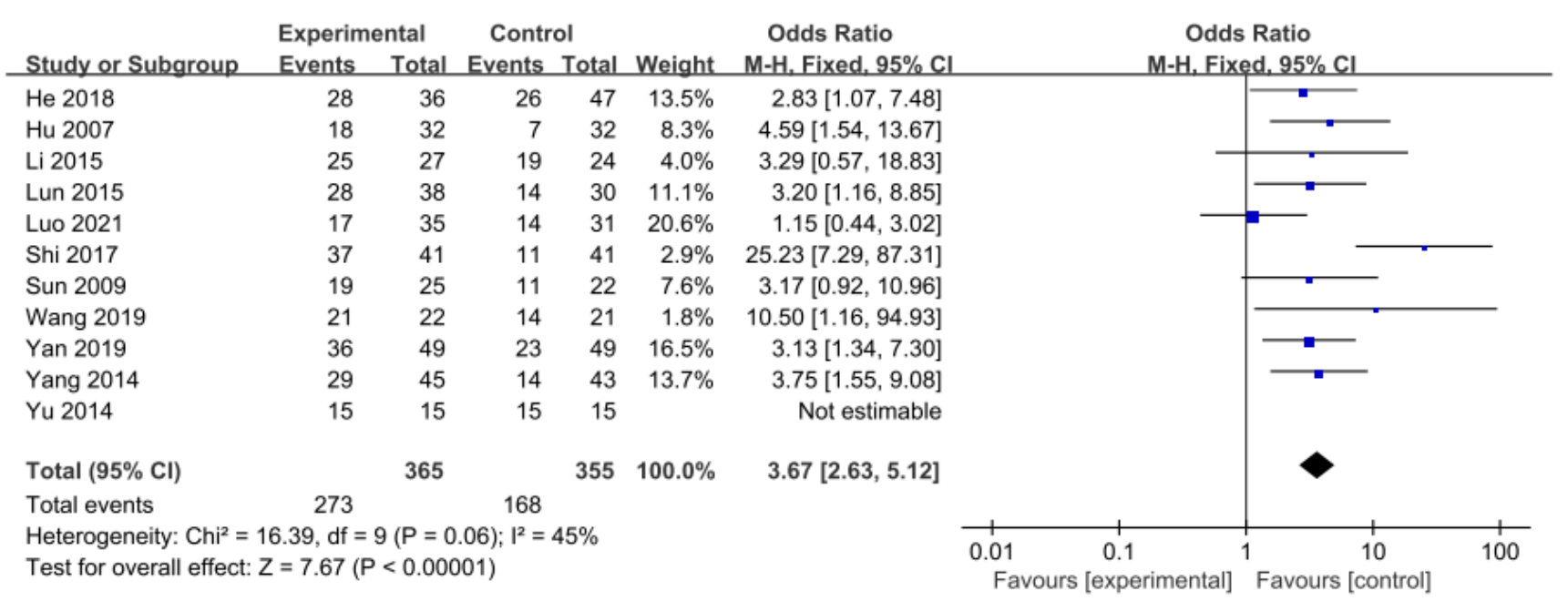

Figure 3: Forest plot of studies evaluating ORs of DCR

Notes: $\mathrm{CI}=$ confidence interval; $\mathrm{OR}=$ odd ratio; $\mathrm{DCR}=$ disease control rate

We performed subgroup analysis including study type (RCT and non RCT), efficacy evaluation time (2 months and 3 months), and chemotherapy regimen (GS regimen and GP regimen). The results of ORR and DCR subgroup analysis are shown in Table 3 and 4, respectively.

Table 3: Results of ORR subgroup analysis

\begin{tabular}{|c|c|c|c|c|c|c|c|}
\hline & Study number & People number & $\mathrm{I}^{2}$ & $\mathrm{Chi}^{2}$ & OR & $95 \% \mathrm{CI}$ & $p$ \\
\hline RCT & 5 & 332 & $24 \%$ & 5.12 & 3.43 & $2.00-5.86$ & $p<0.0001$ \\
\hline non-RCT & 6 & 388 & $21 \%$ & 6.35 & 2.95 & $1.91-4.54$ & $p<0.0001$ \\
\hline GS & 4 & 331 & $0 \%$ & 1.49 & 3.72 & $2.26-6.12$ & $p<0.0001$ \\
\hline GP & 4 & 252 & $41 \%$ & 5.09 & 2.05 & $1.18-3.56$ & $p=0.01$ \\
\hline 2 months & 8 & 519 & $0 \%$ & 3.52 & 4.01 & $2.68-6.01$ & $p<0.0001$ \\
\hline 3 months & 2 & 154 & $11 \%$ & 1.12 & 1.23 & $0.58-2.60$ & $p=0.60$ \\
\hline
\end{tabular}

Table 4: Results of DCR subgroup analysis

\begin{tabular}{|c|c|c|c|c|c|c|c|c|}
\hline & Study number & People number & $\mathrm{I}^{2}$ & $\mathrm{Tau}^{2}$ & $\mathrm{Chi}^{2}$ & OR & $95 \% \mathrm{CI}$ & $p$ \\
\hline RCT & 2 & 332 & $61 \%$ & 0.44 & 7.69 & 5.72 & $2.47-13.24$ & $p<0.0001$ \\
\hline non-RCT & 6 & 388 & $0 \%$ & 0 & 4.67 & 2.58 & $1.63-4.10$ & $p<0.0001$ \\
\hline GS & 4 & 331 & $68 \%$ & 0.56 & 9.42 & 4.81 & $1.97-11.75$ & $p=0.004$ \\
\hline GP & 4 & 252 & $15 \%$ & 0.06 & 3.53 & 2.46 & $1.34-6.07$ & $p<0.0001$ \\
\hline 2 months & 8 & 519 & $41 \%$ & 0.24 & 10.15 & 4.71 & $2.63-8.43$ & $p<0.0001$ \\
\hline 3 months & 2 & 254 & $68 \%$ & 0.48 & 3.14 & 2.11 & $0.66-6.75$ & $p=0.21$ \\
\hline
\end{tabular}

\subsection{Toxicity and Side Effects}

Our meta-analysis included diarrhea, pernicious vomiting, leukopenia, thrombocytopenia, anemia, and hepatic impairment as side events. Because there was no significant statistical difference between the treatment and control groups in terms of side effects: diarrhea: $\mathrm{OR}=0.75,95 \% \mathrm{CI}: 0.42-1.25$; pernicious vomiting: $\mathrm{OR}=1.30,95 \% \mathrm{CI}$ : $0.82-2.06$; leukopenia: $\mathrm{OR}=1.32$, 95\%CI: 0.88-1.98; thrombocytopenia: $\mathrm{OR}=1.05$, 95\%CI: 0.71-1.57; anemia: OR=1.18, 95\%CI: 0.66-2.10; and hepatic impairment: OR=1.10. 95\% CI: 0.63-1.92. (see Figure 4 in the Appendix), we adopted a fixed effects model to assess them. Seed migration and bleeding after puncture only occurred in the treatment group using seed implantation, and there were no serious adverse reactions related to brachytherapy in all studies.

Progression-free survival and Overall survival

For the PFS, three trials were investigated. The pooled HR for PFS was $0.63(95 \%$ CI $0.30-1.32 \mathrm{p}=0.22)$, according to meta-analysis (See Figure 5). The OS was tested on six trails. The pooled HR for OS was $0.72(95 \% \mathrm{CI} 0.43-1.20 \mathrm{p}=0.21)$, according to meta-analysis (See Figure 6). In other words, there was no significant difference between the two groups in terms of PFS and OS.

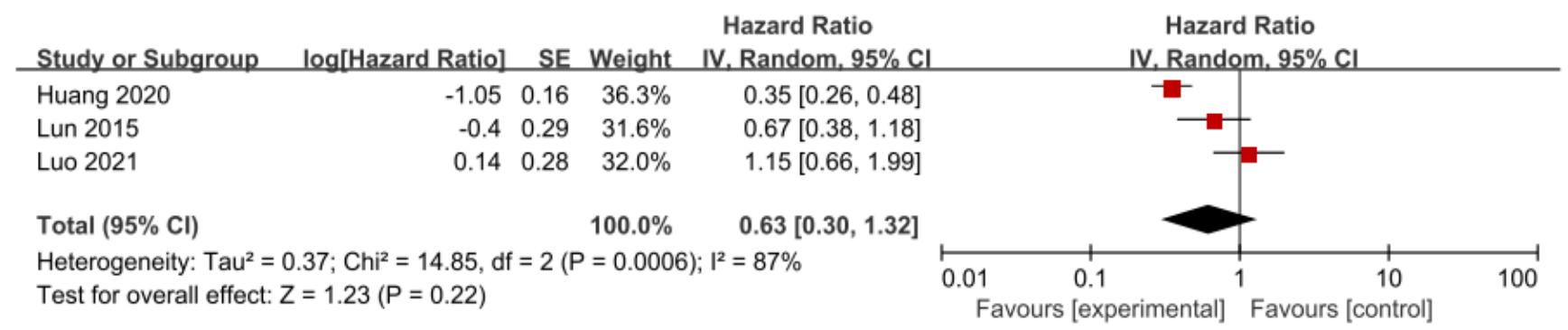

Figure 5: Forest plot for studies evaluating HR of PFS

Notes: $\mathrm{CI}=$ confidence interval; $\mathrm{HR}=$ hazard ratio; $\mathrm{PFS}=$ Progression free survival 


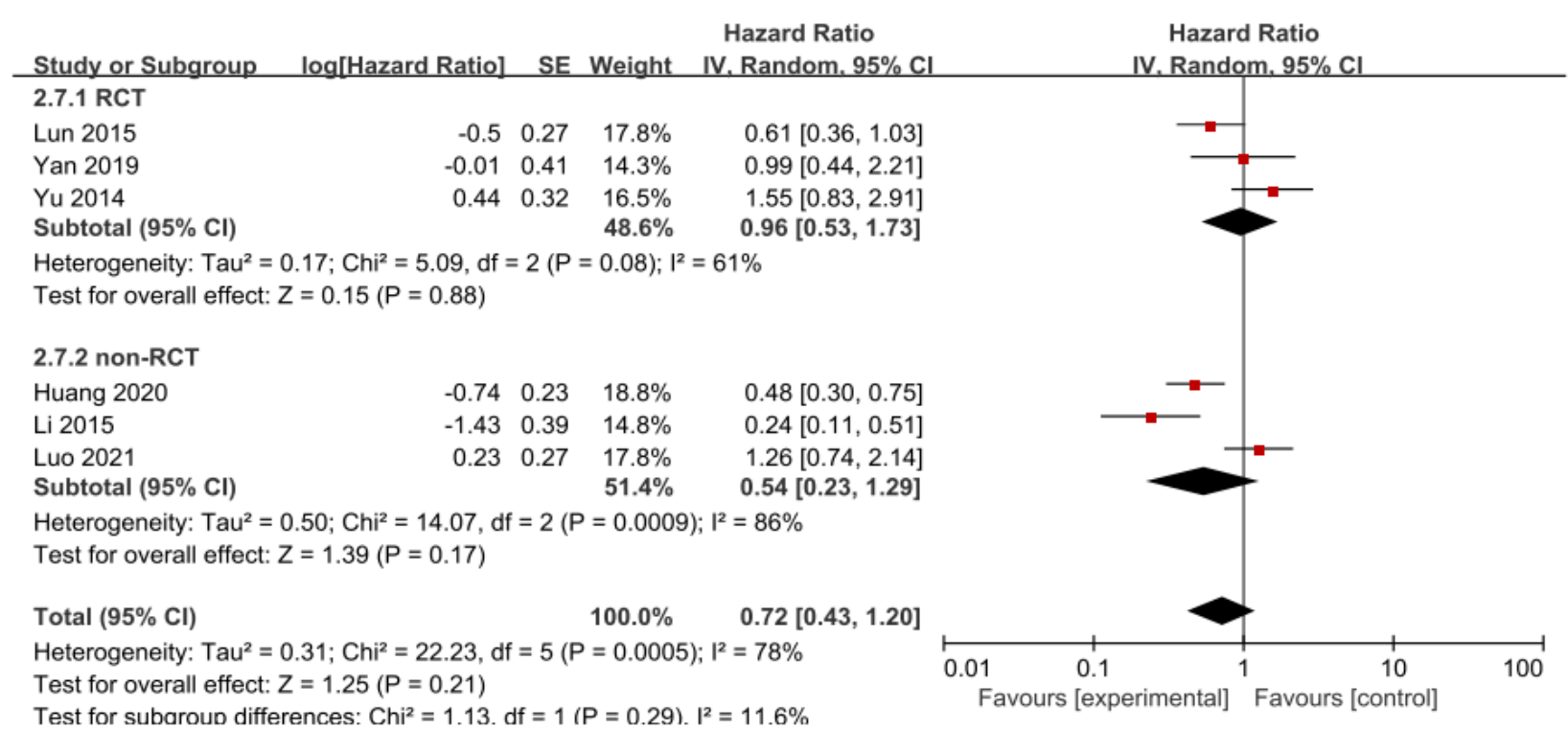

Figure 6: Forest plot for studies evaluating HR of OS

Notes: $\mathrm{CI}=$ confidence interval; $\mathrm{HR}=$ hazard ratio; $\mathrm{OS}=$ overall survival.

\subsection{Publication Bias and Sensitivity Analysis}

The funnel plot in the current meta-analysis revealed that ORR and DCR had no significant publication bias (Figure 7 and Figure 8 ). We performed a sensitivity analysis by deleting one study at a time to estimate the overall results on the ORR, DCR, and OS parameters. After the sensitivity analysis, the pooled estimate of OS remained stable, indicating that the primary findings were not influenced by any particular study (The results are shown in Table 5). Furthermore, using sensitivity analysis, we discovered that omitting Luo's study reduced ORR heterogeneity to $0 \%$, and the aggregate effect size of ORR showed no significant change (OR 3.52, 95\%CI:2.48-5.01, $\mathrm{p}<0.0001)$. Meanwhile, after sensitivity analysis, it was discovered that omitting Shi's study greatly reduced DCR heterogeneity to $0 \%$. The total effect size of DCR, on the other hand, did not vary significantly (OR 3.03, 95\%CI:2.13-4.32, $\mathrm{p}<0.0001)$.

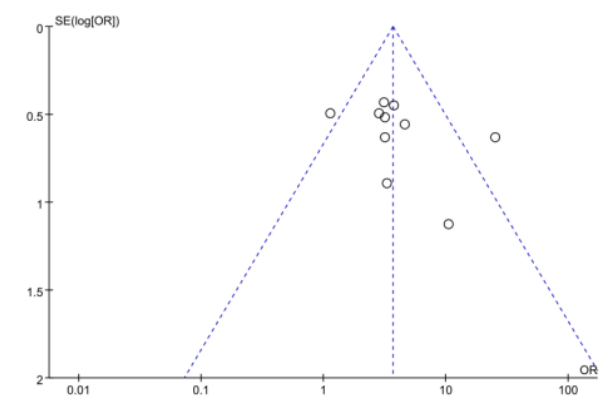

Figure 7: Funnel diagram of studies evaluating ORs of ORR

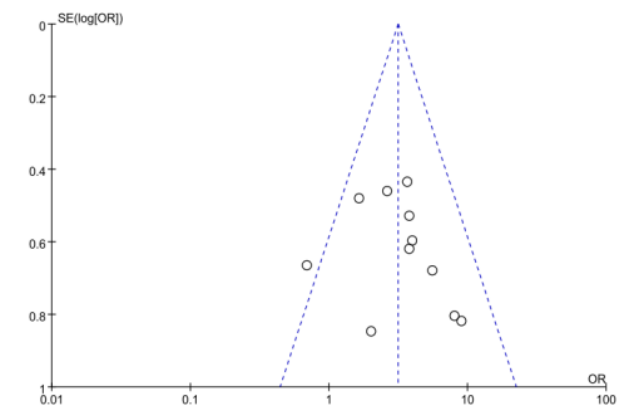

Figure 8: Funnel diagram of studies evaluating ORs of DCR
Table 5: Sensitivity analysis results of OS

\begin{tabular}{ccccccc}
\hline Deleted study & $\mathrm{I}^{2}$ & $\mathrm{Tau}^{2}$ & $\mathrm{Chi}^{2}$ & HR & $95 \% \mathrm{CI}$ & $p$ \\
\hline Huang,2020 & $78 \%$ & 0.36 & 17.91 & 0.79 & $0.43-1.44$ & 0.45 \\
Li,2015 & $70 \%$ & 0.20 & 13.48 & 0.87 & $0.54-1.38$ & 0.55 \\
Lun,2015 & $74 \%$ & 0.25 & 15.43 & 0.62 & $0.37-1.04$ & 0.07 \\
Luo,2020 & $76 \%$ & 0.31 & 16.79 & 0.64 & $0.36-1.12$ & 0.12 \\
Yang.2014 & $82 \%$ & 0.42 & 21.75 & 0.75 & $0.39-1.41$ & 0.37 \\
Yu,2014 & $81 \%$ & 0.36 & 21.55 & 0.68 & $0.38-1.23$ & 0.20 \\
\hline
\end{tabular}

\section{Discussion}

Pancreatic neoplasm is categorised as resectable, borderline resectable, locally advanced or metastatic [31]. Surgical resection is the only treatment that offers a potential cure of pancreatic neoplasm and the definition of resectable pancreatic neoplasm is patients without superior mesenteric artery, celiac axis, portal vein or superior mesenteric vein [32]. With advances in neoadjuvant therapies, a subset of patients with borderline resectable and locally advanced disease have the opportunity for surgery. However, chemotherapy and radiotherapy can lead to intrapancreatic fibrosis, increasing the complication rate associated with pancreatic resection [33]. Even more regrettably, a large proportion of patients are found already to have unresectable pancreatic neoplasm for which no effective treatment is yet available. Firstly, unresectable pancreatic neoplasm is largely absent from the field of immunotherapy's recent success stories. The study by Henriksen A et al showed that in pancreatic neoplasm patients, neither single agent nor dual agent IO, nor combinations with cytotoxic chemotherapy and radiotherapy, among others, have shown meaningful clinical benefit [34]. Thus it can be seen that chemotherapy is the cornerstone of treatment for unresectable pancreatic neoplasm. Despite the fact that cytotoxic chemotherapy is responsible for the greatest survival benefits in the unresectable scenario, new medicines are being researched [35]. In contrast to other data offered regarding the efficacy of chemotherapy in pancreatic neoplasm, there is no substantial evidence for the use of radiation at this time. LAP07, a randomized controlled trial investigating the function of gemcitabine and erlotinib with or without chemoradiotherapy in pancreatic neoplasm, was stopped early because there was no significant difference in survival between chemoradiotherapy and chemotherapy alone 
[36]. In addition, studies have also confirmed that conventional fractionated radiotherapy and chemotherapy up to $60 \mathrm{~Gy}$ can produce moderate local control benefits, but has no obvious effect on survival and is minimal if any [37]. Herman JM et al showed no significant difference in outcomes between the use of SBRT and conventional fractionated chemoradiotherapy for patients with unresectable pancreatic neoplasm [38]. Secondly, although many genes, such as NTRK, BRCA, KRAS, TP53, etc., are mutated in pancreatic neoplasm, there is no therapeutic indication to date [39]. Molecular targeted therapy has largely failed to improve the survival of pancreatic neoplasm patients [40]. Therefore, a reality that must now be faced is that the magnitude of pancreatic neoplasm urgently requires therapeutic innovation in combination with conventional chemotherapy. Iodine-125 ${ }^{125}$ I) seed implantation is a type of brachytherapy or interventional radiotherapy that has been used in the treatment of pancreatic neoplasm for decades. Because ${ }^{125}$ I seeds can be placed directly within the tumor and have a small radiation radius, a high radioactive dose can be used to kill nearby tumor cells without causing significant collateral damage to surrounding tissues [41]. Pancreatitis, fistulas, seed migration, ulcers, infections, leaks, and intestinal perforations are the most common (significant) consequences of percutaneous ${ }^{125} \mathrm{I}$ seed implantation [42]. To date, no major or fatal side effects have been documented. The use of seeds in the treatment of unresectable pancreatic neoplasms was shown to suppress local tumor growth and extend the survival of patients with stage II and III pancreatic neoplasm to some extent without causing further difficulties, with a median survival time of 10.1 months [43].

Twelve clinical trials were included in this review. There were 863 patients with stage II-IV pancreatic neoplasms, ranging in age from 29 to 83 years, ensuring a large enough sample size for meta-analysis. We believe the research we included are of high quality, given the uniqueness of the treatment and the difficulties of concealing allocation and implementing blinding, and the methodological quality and sample size were consistent across most included studies. As a result, the outcomes are objective and consistent. In treating unresectable pancreatic neoplasm, ${ }^{125} \mathrm{I}$ brachytherapy combined with chemotherapy improved ORR and DCR, according to our meta-analysis. The ORR and DCR of ${ }^{125} \mathrm{I}$ brachytherapy combined with gemcitabine-containing chemotherapy to treat unresectable pancreatic neoplasm were compared, and the results revealed a significant difference between the treatment and control groups. In patients with stage II/III/IV pancreatic neoplasms, ${ }^{125} \mathrm{I}$ brachytherapy paired with treatment with gemcitabine could considerably improve ORR and DCR. These findings are similar to those of prior retrospective research [44], but we included a larger number of trials to increase statistical reliability. The publication bias test revealed that neither the ORR nor the DCR results had any clear publication bias. In conclusion, the short-term efficacy of ${ }^{125}$ I seed implantation combined with chemotherapy, namely ORR and DCR, is better than chemotherapy alone. This may be due to the $\gamma$ - ray generated by ${ }^{125} \mathrm{I}$ seeds, which can destroy the DNA double strand of tumor cells at a low dose and at the same time delay the cycle progression of already proliferating malignant tumor cells, thereby inhibiting tumor cell proliferation, inducing tumor cell apoptosis, and inhibiting tumor cell angiogenesis and it can destroy the retroperitoneal plexus and reduce pain as well [45-46]. What's more, gemcitabine, a chemotherapeutic drug included in these studies, is a pyrimidine nucleotide analog that inhibits DNA synthesis and promotes apoptosis of tumor cells [47]. Both ${ }^{125}$ I seeds and gemcitabine act on the DNA synthesis process of tumor cells, and ${ }^{125} \mathrm{I}$ seeds can arrest tumor cells in the G2 / M phase [48], while gemcitabine blocks the cell cycle process at the G1/S phase boundary [49]. Both modalities work together on tumor cells, therefore the combination treatment group's current efficacy is much better than the chemotherapy alone group's. Unfortunately, there was no statistically significant difference in OS and PFS in this trial. The addition of local therapy was not effective in prolonging survival, and this reason is probably multifactorial and may be largely influenced by the high metastatic rate of pancreatic neoplasm. Moreover, the included literatures did not all explicitly give the dose of internal radiotherapy, and insufficient dose may also be one of the reasons for no difference in survival. As a result, investigations with a longer follow-up time are needed in the future to determine the long-term survival of patients with unresectable pancreatic neoplasm treated with ${ }^{125} \mathrm{I}$ brachytherapy combined with chemotherapy.

There was no statistically significant difference in side effects between the combination therapy and chemotherapy groups, indicating that ${ }^{125} \mathrm{I}$ brachytherapy combined with chemotherapy does not enhance the risk of common adverse responses. The primary adverse effects found in both the treatment and control groups in the papers we extracted are diarrhea, pernicious vomiting, leukopenia, thrombocytopenia, anemia and hepatic impairment. The reason is that gastrointestinal symptoms (diarrhea and pernicious vomiting), leukopenia, myelosuppression (thrombocytopenia and anemia) and hepatic impairment are mainly caused by chemotherapy [50-51]. Some complications including pancreatic fistula, pancreatitis, seed migration, and duodenitis appeared in the group that received ${ }^{125} \mathrm{I}$ treatment, but no severe, potentially lethal complications related to seed implantation occurred in all included studies. This results from the characteristics of ${ }^{125}$ I seed. The half-life of ${ }^{125}$ I seed is 59.7 days, which is ideal for pancreatic neoplasms that grow quickly [52]. The average photon energy in a ${ }^{125} \mathrm{I}$ seed is $27-35 \mathrm{keV}$ gamma rays, the initial release is $7 \mathrm{cGy} / \mathrm{h}$, the average radiation is $0.694 \pm$ $0.021 \mathrm{mCi}(25.6 \mathrm{MBq})$ and the penetration hyperintensity in human tissues is only $1.7 \mathrm{~cm}$ [53]. Therefore, ${ }^{125} \mathrm{I}$ seed implantation can make the interior energy of the tumor high without irradiating the surrounding organs, and similarly, it is harmless to the patient's relatives as long as protection is made. At the same time, the hazards to the operators can be minimized by adequate shielding.

There were various flaws in this meta-analysis. To begin, the meta-analyses cover both RCTs and non-RCTs. Because non-RCTs are not randomized, it is difficult to confirm that the two treatment groups are comparable before treatment. Second, multiple chemotherapy regimens, cycles, and doses of chemotherapy were used in different studies, and no single dosage of radiation therapy was used, which could lead to bias in the study. Third, while examining complications resulting from ${ }^{125}$ I seed implantation, there was only a small number of patients, which may have resulted in an insufficient assessment of the safety of ${ }^{125} \mathrm{I}$ brachytherapy. We adopted a 
tight inclusion criterion to select high-quality trials during the literature filtering procedure to assure the correctness of the pooled data. Because the characteristics of minimally invasive treatment make a totally random allocation and blind technique difficult [54], we chose clinical trials with reduced risk after evaluating Cochrane ROB criteria. To reduce selection bias, all of the non-RCT studies included were meant to be as near to RCTs as possible in methodology and had comparability between the two groups. We pooled our results independently according to different treatment approaches during the analytical procedure. To establish the consistency of our findings, we used subgroup analysis and sensitivity analysis. Due to the large heterogeneity between various studies, we use stochastic effect models to merge to draw more cautious conclusion. We hope that our data will support the efficacy and safety of ${ }^{125}$ I brachytherapy in combination with gemcitabine-containing chemotherapy.

\section{Conclusion}

${ }^{125}$ I brachytherapy combined with the inclusion of gemcitabine chemotherapy enhance the clinical efficacy of patients with unresectable pancreatic neoplasm without increasing the incidence of adverse effects such as pancreatic fistula, pancreatitis, seed migration, and duodenitis compared with chemotherapy. However, there was no effect on the survival of unresectable pancreatic neoplasm.

\section{Disclosure}

The authors report no proprietary or commercial interest in any product mentioned or concept discussed in this article.

\section{Acknowledgments}

AXS. and LYY. designed research process; LYY. and SY. screened and reviewed all literature that conformed to the inclusion criteria and completed statistical analysis of the data; LYY, HUC, QMZ and YYL interpreted the outcomes; AXS made the decision to submit the article for publication.

\section{References}

[1] Siegel RL, Miller KD, Jemal A. Cancer statistics, 2020. CA Cancer J Clin 2020; 70:7-30. DOI:10.3322/caac.21590.

[2] Chen W, Zheng R, Baade PD, et al. Cancer statistics in China, 2015. CA Cancer J Clin 2016; 66:115-132. DOI:10.3322/caac.21338.

[3] Kamisawa T, Wood LD, Itoi T, et al. Pancreatic neoplasm. Lancet. 2016; 388:73-85. DOI:10.1016/S0140-6736 (16)00141-0.

[4] Zeng S, Pöttler M, Lan B, et al. Chemoresistance in Pancreatic neoplasm. Int J Mol Sci. 2019 Sep 11; 20 (18):4504. DOI:10.3390/ijms20184504.

[5] Bosetti C, Bertuccio P, Negri E, et al. Pancreatic neoplasm:overview of descriptive epidemiology. Mol Carcinog. 2012; 51 (1):3-13. DOI:10.1002/mc.20785.

[6] Oberstein PE, Olive KP. Pancreatic neoplasm:why is it so hard to treat? Therap Adv Gastroenterol. 2013; 6 (4):321-337. DOI:10.1177/1756283X13478680.
[7] Lambe M, Eloranta S, Wigertz A, et al. Pancreatic neoplasm; reporting and long-term survival in Sweden.

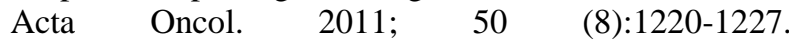
DOI:10.3109/0284186X.2011.599338.

[8] Zhang QH, Ni QX; Coordination Group of The Committee on Pancreatic neoplasm. [Clinical analysis of 2340 cases of pancreatic neoplasm]. Zhonghua Yi Xue Za Zhi. 2004 Feb 2; 84 (3):214-8. Chinese. PMID:15059537.

[9] Kleeff J, Korc M, Apte M, et al. Pancreatic neoplasm. Nat Rev Dis Primers. 2016 Apr 21; 2:16022. DOI:10.1038/nrdp.2016.22.

[10] Mizrahi JD, Surana R, Valle JW, et al. Pancreatic neoplasm. Lancet. 2020 Jun 27; 395 (10242):2008-2020. DOI:10.1016/S0140-6736 (20)30974-0.

[11] Peretz T, Nori D, Hilaris B, et al. Treatment of primary unresectable carcinoma of the pancreas with I-125 implantation. Int J Radiat Oncol Biol Phys. 1989 Nov; 17 (5):931-5. DOI:10.1016/0360-3016 (89)90138-7.

[12] Chin V, Nagrial A, Sjoquist K, et al. Chemotherapy and radiotherapy for advanced pancreatic neoplasm. Cochrane Database Syst Rev. 2018 Mar 20; 3 (3):CD011044. DOI:10.1002/14651858.

[13] Moher D, Liberati A, Tetzlaff J, et al. Preferred reporting items for systematic reviews and meta-analyses: the PRISMA statement. PLoS Med. 2009 Jul 21; 6 (7): e1000097. DOI: 10.1371/journal.pmed.1000097.

[14] Higgins JP, Altman DG, Gøtzsche PC, et al. Cochrane Bias Methods Group; Cochrane Statistical Methods Group. The Cochrane Collaboration's tool for assessing risk of bias in randomised trials. BMJ. 2011 Oct 18; 343: d5928. DOI:10.1136/bmj. d5928.

[15] Margulis AV, Pladevall M, Riera-Guardia N, et al. Quality assessment of observational studies in a drug-safety systematic review, comparison of two tools:the Newcastle-Ottawa Scale and the RTI item bank. Clin Epidemiol. 2014; 6:359-368. Published 2014 Oct 10. DOI: $10.2147 /$ CLEP.S66677

[16] Duffaud F, Therasse P. Nouvelles recommandations pour l'évaluation de la réponse tumorale dans les tumeurs solides [New guidelines to evaluate the response to treatment in solid tumors]. Bull Cancer. 2000 Dec; 87 (12):881-6. French. PMID:11174117.

[17] Tierney JF, Stewart LA, Ghersi D, et al. Practical methods for incorporating summary time-to-event data into meta-analysis. Trials. 2007 Jun 7; 8:16 DOI:10.1186/1745-6215-8-16.

[18] Sterne JA, Sutton AJ, Ioannidis JP, Terrin N, et al. Recommendations for examining and interpreting funnel plot asymmetry in meta-analyses of randomised controlled trials. BMJ. 2011 Jul 22; 343: d4002. DOI:10.1136/bmj. d4002.

[19] Yu YP, Yu Q, Guo JM, et al. (125) I particle implantation combined with chemoradiotherapy to treat advanced pancreatic cancer. Br J Radiol. 2014 Apr; 87 (1036):20130641. DOI:10.1259/bjr.20130641.

[20] Shi Guangyong. Clinical Evaluation of CT Guided 125I Particle Implantation Combined with Topical Drugs Chemotherapy. China Pharmaceuticals. 2017, 14:50-53. DOI: 10.3969/j.issn.1006-4931.2017.14.018

[21] Yang WK, Chen Y, Wang L, et al. CT-guided 125I seed interstitial implantation combined with gemcitabine/cisplatin chemotherapy for treatment of 
locally advanced pancreatic carcinoma. World Chinese Journal of Digestology. 2014, 10:1455-1459. DOI: CNKI: SUN: XXHB.0.2014-10-024

[22] Hu ZQ, Qian LY, Wei YS, et al. 125I radioactive particles implanted into tumor combind with gemcitabine to treat extended local pancreas cancer[J]. China Medical Engineering. 2007, 015 (001):87-89, 92. DOI: 10.3969/j.issn.1672-2019.2007.01.032

[23] Luo M, Chen J, Zhong Z, et al. CT-guided 125I brachytherapy combined with chemotherapy for the treatment of unresectable or locally advanced pancreatic carcinoma. Diagn Interv Radiol. 2021 Jan; 27 (1):50-58. DOI:10.5152/dir.2020.19371.

[24] Yan BJ, Han NN. Effect of CT guided (125) I radioactive seed implantation combined with GS chemotherapy on disease control rate and median progression free survival in patients with advanced pancreatic cancer[J]. Capital Food Medicine.2019, 26 (09):27-27. DOI: CNKI: SUN: YYSD.0.2019-09-021

[25] Wang YL, Wang ZH. Clinical efficacy of CT-guided 125I particle implantation combined with gemcitabine and capecitabine in treatment of locally advanced pancreatic cancer[J]. Shaanxi Medical Journal. 2020,49 (12):1604-1607.

DOI: 10.3969/j.issn.1000-7377.2020.12.017

[26] Sun Y, Gao JB, Zhou ZG, et al. CT guided (125) I seed implantation combined with chemotherapy for 25 patients with advanced pancreatic cancer: [J]. Journal of Zhengzhou University (Medical Sciences). 2009, 44 (6): $1273-1274$.

DOI: 10.3969/j.issn.1671-6825.2009.06.052

[27] LI Shuai, ZHOU Zhigang, GAO Jianbo, et al. Radioactive I seeds implantation combined with gemcitabine plus cisplatin treatment of pancreatic cancer. [J]. Journal of Practical Radiology. 2015, 31 (011):1839-1841, $1875 . \quad$ DOI: 10.3969/j.issn.1002-1671.2015.11.023

[28] Huang SQ, Cui CJ, Ji SC, et al. Clinical effectiveness of 125I radioactive particle implantation combined with chemotherapy in the treatment for unresectable advanced pancreatic carcinoma[J]. Chinese Journal of Cancer Prevention and Treatment. 2020,27 (17), 1415-1420. DOI: 10.16073/j.cnki.cjcpt.2020.17.10

[29] He AL. The clinical value of CT guiding 125I radioactive particle implantation combined with GS chemotherapy in the treatment of advanced pancreatic cancer[J]. Ningxia Medical Journal,2018,40 (08):51-53. DOI:10.13621/j.1001-5949.2018.08.0717

[30] Lun JJ, Zhao JL, Sun JY, et al. CT- guided 125 I radioactive seed interstitial implantation combined with chemotherapy for advanced pancreatic carcinoma: analysis of therapeutic efficacy [J]. Journal of Interventional Radiology, 2015 (06):494-497. DOI: 10.3969/j.issn.1008-794X.2015.06.007

[31] Loveday BPT, Lipton L, Thomson BN. Pancreatic neoplasm:An update on diagnosis and management. Aust J Gen Pract. 2019 Dec; 48 (12):826-831. DOI:10.31128/AJGP-06-19-4957.

[32] McGuigan A, Kelly P, Turkington RC, et al. Pancreatic neoplasm:A review of clinical diagnosis, epidemiology, treatment and outcomes. World J Gastroenterol. 2018 Nov 21; 24 (43):4846-4861. DOI:10.3748/wjg. v24. i43.4846.
[33] Lopez NE, Prendergast C, Lowy AM. Borderline resectable pancreatic neoplasm:definitions and management. World J Gastroenterol. 2014 Aug 21; 20 (31):10740-51. DOI:10.3748/wjg. v20. i31.10740.

[34] Henriksen A, Dyhl-Polk A, Chen I, Nielsen D. Checkpoint inhibitors in pancreatic neoplasm. Cancer Treat Rev. 2019 Aug; 78:17-30. DOI: 10.1016/j.ctrv.2019.06.005.

[35] Roth MT, Cardin DB, Berlin JD. Recent advances in the treatment of pancreatic neoplasm. F1000Res. 2020 Feb 21; 9: F1000 Faculty Rev-131. DOI:10.12688/f1000research.21981.1.

[36] Hammel P, Huguet F, van Laethem JL, et al. LAP07 Trial Group. Effect of Chemoradiotherapy vs Chemotherapy on Survival in Patients with Locally Advanced Pancreatic neoplasm Controlled After 4 Months of Gemcitabine With or Without Erlotinib: The LAP07 Randomized Clinical Trial. JAMA. 2016 May 3; 315 (17):1844-53. DOI:10.1001/jama.2016.4324.

[37] Reyngold M, Parikh P, Crane $\mathrm{CH}$. Ablative radiation therapy for locally advanced pancreatic neoplasm: techniques and results. Radiat Oncol. 2019 Jun 6; 14 (1):95. DOI:10.1186/s13014-019-1309-x.

[38] Herman JM, Chang DT, Goodman KA, et al. Phase 2 multi-institutional trial evaluating gemcitabine and stereotactic body radiotherapy for patients with locally advanced unresectable pancreatic adenocarcinoma.

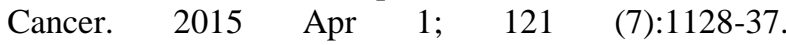
DOI:10.1002/cncr.29161.

[39] Biankin AV, Waddell N, Kassahn KS, et al. Pancreatic neoplasm genomes reveal aberrations in axon guidance pathway genes. Nature. 2012 Nov 15; 491 (7424):399-405. DOI:10.1038/nature11547.

[40] Ryan DP, Hong TS, Bardeesy N. Pancreatic adenocarcinoma. N Engl J Med. 2014 Nov 27; 371 (22):2140-1. DOI:10.1056/NEJMc1412266.

[41] Timmer FEF, Geboers B, Nieuwenhuizen S, et al. Locally Advanced Pancreatic neoplasm: Percutaneous Management Using Ablation, Brachytherapy, Intra-arterial Chemotherapy, and Intra-tumoral Immunotherapy. Curr Oncol Rep. 2021 Apr 17; 23 (6):68. DOI:10.1007/s11912-021-01057-3.

[42] Lv WF, Lu D, Xiao JK, Mukhiya G, Tan ZX, Cheng DL, Zhou CZ, Zhang XM, Zhang ZF, Hou CL. The side effects and complications of percutaneous iodine-125 seeds implantation under CT-guide for patients with advanced pancreatic neoplasm. Medicine (Baltimore). 2017 Dec; $96 \quad$ (52): DOI:10.1097/MD.0000000000009535.

[43] Wang H, Wang J, Jiang Y, et al. The investigation of 125I seed implantation as a salvage modality for unresectable pancreatic carcinoma. J Exp Clin Cancer Res. 2013; 32 (1):106. Published 2013 Dec 27. DOI:10.1186/1756-9966-32-106

[44] Ning Z, Li H, Guo X, Cui Z, Qi X, et al. Curative effect of I-125 particles implantation combined with chemotherapy and chemotherapy alone in pancreatic neoplasm:a Meta-analysis. Chinese Journal of Gastroenterology and Hepatology. 2016 Mar; 25 (03):320-325. DOI: CNKI: SUN: WCBX.0.2016-03-026. 
[45] GuX Z, Yin WB, Yu ZH. Radiation oncology[M]. Beijing:Pecking Union Medical College Press, 2015:222-284.

[46] Luo KY. Practical interstitial brachytherapy of malignant tumor[M]. Beijing: People's Health Publishing House, 2008:39-45.

[47] Zeng S, Pöttler M, Lan B, et al. Chemoresistance in Pancreatic neoplasm. Int J Mol Sci. 2019 Sep 11; 20 (18):4504. DOI:10.3390/ijms20184504.

[48] Wang J, Wang J, Liao A, et al. The direct biologic effects of radioactive 125I seeds on pancreatic neoplasm cells PANC-1, at continuous low-dose rates. Cancer Biother Radiopharm. 2009 Aug; 24 (4):409-16. DOI:10.1089/cbr.2008.0563.

[49] Zeng S, Pöttler M, Lan B, et al. Chemoresistance in Pancreatic neoplasm. Int J Mol Sci. 2019; 20 (18):4504. Published 2019 Sep 11. DOI:10.3390/ijms20184504

[50] Ayhanci, A., Günes, S., Sahinturk, V. et al. Seleno L-Methionine Acts on Cyclophosphamide-Induced Kidney Toxicity. Biol Trace Elem Res 136, 171-179 (2010). DOI:10.1007/s12011-009-8535-2
[51] Yang Y, Xu S, Xu Q, et al. Protective effect of Dammarane Sapogenins against chemotherapy-induced myelosuppression in mice. Experimental Biology and
Medicine.
2011;
236
(6):729-735.

DOI:10.1258/ebm.2011.010369

[52] Han Q, Deng M, Lv Y, et al. Survival of patients with advanced pancreatic neoplasm after iodine125 seeds implantation brachytherapy:A meta-analysis. Medicine $\begin{array}{lllll}\text { (Baltimore). } 2017 & \text { Feb; } 96 & \text { (5): } \quad \text { e5719. }\end{array}$ DOI:10.1097/MD.0000000000005719.

[53] Du YQ, Li ZS, Jin ZD. Endoscope-assisted brachytherapy for pancreatic neoplasm:From tumor killing to pain relief and drainage. J Interv Gastroenterol. 2011 Jan; 1 (1):23-27. DOI:10.4161/jig.1.1.14596.

[54] Stewart A, Parashar B, Patel M, et al. American Brachytherapy Society consensus guidelines for thoracic brachytherapy for lung cancer. Brachytherapy. 2016 Jan-Feb; $\quad 15$ (1):1-11. DOI: 10.1016/j.brachy.2015.09.006. 


\section{Appendix}



Figure 4: Forest plot for the comparison of toxicity and adverse reactions (the treatment groups) Notes: $\mathrm{CI}=$ confidence interval; $\mathrm{OR}=$ odd ratio. 